\title{
Determination of the average ionization and thermodynamic regimes of xenon plasmas with an application to the characterization of blast waves launched in xenon clusters
}

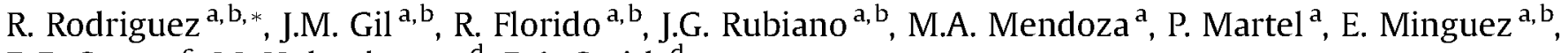 \\ D.R. Symes ${ }^{c}$, M. Hohenberger ${ }^{d}$, R.A. Smith ${ }^{d}$ \\ ${ }^{a}$ Departmento de Fisica, Universidad de Las Palmas de Gran Canaria, Campus de Tafira, 35017 Las Palmas de Gran Canaria, Spain \\ ${ }^{\mathrm{b}}$ Instituto de Fusión Nuclear, Universidad Politécnica de Madrid, 28006 Madrid, Spain \\ ${ }^{\mathrm{c}}$ Central Laser Facility, Rutherford Appleton Laboratory, Oxfordshire OX11 OQX, UK \\ 'Blackett Laboratory, Imperial College, London SW7 2BZ, UK
}

Keywords:

Xenon plasmas

Thermodynamic regimes

Average ionization

Radiative blast waves

\begin{abstract}
A B S T R A C T
Radiative shock waves play a pivotal role in the transport energy into the stellar medium. This fact has led to many efforts to scale the astrophysical phenomena to accessible laboratory conditions and their study has been highlighted as an area requiring further experimental investigations. Low density material with high atomic mass is suitable to achieve radiative regime, and, therefore, low density xenon gas is commonly used for the medium in which the radiative shock propagates. In this work the average ionization and the thermodynamic regimes of xenon plasmas are determined as functions of the matter density and temperature in a wide range of plasma conditions. The results obtained will be applied to characterize blast waves launched in xenon clusters.
\end{abstract}

\section{Introduction}

Shock waves are ubiquitous throughout the universe and play a crucial role in the transport of energy into the interstellar medium [1]. Therefore, the understanding of the dynamics and evolution of shock waves is crucial to the understanding of the structure of the interstellar medium [2]. When the radiation transport is important to the total energy budget, shock waves can be radiatively driven so that its dynamics can be significantly modified by radiative processes. At high shock velocity, the shocked medium is heated and ionized emitting radiation, which gives rise to radiative cooling. The radiation emitted in turn heats and ionizes the unshocked medium leading to the creation of a radiative precursor [3]. Radiative shocks are observed around astronomical objects in a wide variety of forms, e.g. accretion shock, pulsating stars, supernovae in their radiative cooling stage, bow shocks of stellar jet in galactic medium, collision of interstellar clouds and entry of rockets or comets into planetary atmospheres [4-7] and they are also observed in laser inertial fusion [8]. However, each astronomical observation is unique and predominantly provides information on a small part of the shock evolution. This fact has led many groups to scale these astrophysical phenomena to accessible laboratory conditions and, recently, the study of radiative shocks has been highlighted as an area requiring further experimental investigations [1]. Moreover, laboratory studies of radiative shocks are also motivated by the developing ability to perform radiation-hydrodynamic experiments and by the need for experiments to benchmark new radiation-hydrodynamics codes useful for astrophysical simulations [3]. For a given shock velocity and a given initial gas pressure, materials with high atomic numbers suit the achievement of the radiative regime, and, for this reason, xenon is commonly employed for the medium in which the radiative shock propagates [9-14].

The need for accurate computation of radiative properties covers an extensive range of thermodynamic conditions. Depending on the thermodynamic character of the plasma under analysis (local thermodynamic equilibrium, LTE, or non local thermodynamic equilibrium, NLTE) existing physics models have different uses or implementations [15]. Under LTE conditions there exists an equation that gives the charge state distribution and the occupation probabilities of bound states, i.e., the Saha-Boltzmann (SB) equation. On the other hand, under NLTE conditions, the problem is complicated because there is not a priori expression for the 
occupation probabilities of bound states and therefore one must find the distribution of the ionic states by using a collisional-radiative model (CRM) [16] which requires the solution of a set of rate equations, with coupling of configurations, free electrons and photons. Moreover, if we consider that for accurate simulations of the emission and the absorption spectra it is essential to take into account as many quantum levels required to provide an accurate solution, the calculations can become computationally large. The number of the atomic levels involved, and therefore the amount of atomic data required is large and approximations must be made. For low and intermediate Z-plasmas Detailed Level Accounting (DLA) or Detailed Term Accounting (DTA) models are commonly used. On the other hand, for high-Z elements statistical approaches involving grouping of levels such as the Detailed Configuration Accounting (DCA) approach or SuperConfiguration Accounting (SCA) approach $[17,18]$ have been shown to be very efficient when they are combined with Unresolved Transition Array (UTA/SOSA) [19] and/or the SuperTransition Array (STA) formalisms [20]. More recently, new hybrid models that mix detailed levels and configurations are under development [21-23]. For these reasons, NLTE calculations are more difficult than LTE calculations.

The first objective of this paper is to determine for a range of plasma temperatures and densities, the average ionization state of the xenon plasmas and assess whether the plasma is in LTE or not. Whether a plasma is in LTE or not is important for several reasons: First, this allows us to establish in which density and temperature regions the LTE assumption is accurate. Second, the map for the average ionization identifies which are the most abundant ions for each plasma conditions. This fact will allow one to optimize subsequent computations of the radiative properties since the number of ions included in the calculation is reduced which is very relevant in elements like xenon with moderately high atomic number. Furthermore, this reduction allows us to include more atomic configurations per ion, which is important in the determination of the plasma atomic level populations and, therefore, of the radiative properties.

The second objective of this work is to characterize typical blast waves launched in xenon clusters [12,14]. A blast wave is generally described as an expanding shock wave that is in the process of sweeping up the material that is ahead of the shock, which is important in astrophysical scenarios such as the supernova remnants. Blast waves launched in clusters typically have a gas density $\approx 5-10^{-4} \mathrm{gcm}^{-3}$ and post-shock temperatures $\approx 5-10 \mathrm{eV}$. The conditions indicate that these blast waves are well within the radiative flux regime as the radiative energy flux is greater than the material energy flux, but well below the radiation dominated regime, in which the radiation pressure exceeds the material pressure [14]. The use of Xe has an advantage over experiments with solid plastic or foam targets, because at modest laser energies of $<1 \mathrm{~J}$ one can access the radiative flux regime with shock velocities of $\approx 10 \mathrm{kms}^{-1}$. In this work we are interested in probing blast waves launched in xenon clusters belonging to the thin-thin regime of the radiative shocks [24], where the blast wave is actually radiative and, finally, in determining the thermodynamic regime of pre- and post-shock regions. All the calculations presented in this work were performed using the computational package ABAKO/ RAPCAL [25]. Section 2 is devoted to a brief explanation of both codes. In sections 3 and 4 , results and main conclusions are presented, respectively.

\section{Theoretical model}

The calculations in this work were performed using the computational package ABAKO/RAPCAL [25] that consists of two codes, ABAKO [26] and RAPCAL [27].

\section{1. $A B A K O$}

ABAKO is devoted to the calculation of the plasma level populations for arbitrary optical depths in both LTE and NLTE conditions using a collisional-radiative steady state (CRSS) model. The CRSS model is solved level by level (or configuration by configuration, depending on the DLA or DCA approach) and it is applied to low-tohigh $Z$ ions under a wide range of plasma conditions: Coronal Equilibrium, NLTE or LTE, optically thin and thick plasmas. Special care was taken during the development of our CRSS model to achieve an optimal balance between accuracy and computational cost. Hence, analytical expressions have been employed for the rate coefficients of the atomic processes included in the CRSS model, which yield a substantial saving of computational requirements, but still providing satisfactory results in relation to those obtained from more sophisticated codes and experimental data as it has been shown in recent NLTE code comparison workshops [28-30]. The processes included in the CRSS model are the following: collisional ionization [31] and three-body recombination, spontaneous decay, collisional excitation [32] and deexcitation, radiative recombination [33], electron capture and autoionization. We have added between brackets the references wherefrom their approximated analytical rates coefficients have been acquired. The rates of the inverse processes are obtained through the detailed balance principle. It is worth pointing out that the autoionizing states are included explicitly. It has been shown that these contributions are critical to the determination of the ionization balance. The cross section of the autoionization is evaluated using detailed balance principle from the electron capture cross section which is obtained from the collisional excitation cross section using the approximation given in Ref. [34].

Since the number of rate equations is large due to the number of atomic levels involved, ABAKO makes use of the technique of sparse matrices to storage the non-zero elements of the coefficient matrix of the system, which implies substantial savings in memory requirement. For the matrix inversion we use iterative procedures [25] because they entail much less memory than direct methods and, importantly, are faster.

The atomic data employed in this work were obtained from FAC code [35]. The calculations were carried out in the DCA approach. The radiative transitions rates in FAC are calculated in the single multipole approximation, and in this work they were obtained in the electric dipole approach. The UTA [19] formalism is used for the bound-bound transitions, so the transition energies include the UTA shift and width for each transition is provided. Furthermore, the line strengths are corrected for configuration interaction within the same non-relativistic configurations. The continuum lowering due to the influence of the plasma surrounding is also considered and is calculated by means of the expression due to Stewart and Pyatt [36]. Due to the inclusion of the continuum lowering, the kinetics equations must be solved iteratively, since the atomic data depend on ionization balance.

A key factor in the CRM calculations is the choice of configurations included in the model. Including configurations with energies up to three times the ionization potential should be adequate for accurate modeling of thermal plasmas $[22,37]$. Thus, the set of configurations included was selected according to the following criteria:(1) ground configuration; (2) single excited configurations from the valence shell, $n_{v}$, to shells with $n_{v} \leq n \leq n_{v}+4$; (3) doubly-excited configurations from the valence shell to shells with $n_{v} \leq n \leq n_{v}+2$ and (4) single excited configurations from the shell $n_{v}-1$ to shells with $n_{v} \leq n \leq n_{v}+2$.

\subsection{RAPCAL}

RAPCAL code determines several relevant plasma radiative properties such as the spectrally resolved opacities and emissivities, 
mean and multigroup opacities, source functions, radiative power losses, specific intensities and plasma transmission. A detailed description of the code can be found in Ref. [27]. For this work, we focus our interest on the multifrequency and mean opacities as well as radiative power losses. For line transitions, natural, Doppler, UTA and electron-impact [38] broadenings were included. The lineshape function is applied by use of a Voigt profile that incorporates all these broadenings. In this work we have assumed complete redistribution hypothesis and, therefore, we have taken the same profiles for emission and absorption. Since the line contribution is dominant for the plasma conditions analyzed, photoionization cross sections have been evaluated using the semiclassical expression of Kramers [33].

\section{Results}

\subsection{Study of the thermodynamic regimes}

We have obtained the map of the average ionization as a function of matter density and electron temperature (see Fig. 1). The range of plasma conditions considered covers both LTE and NLTE conditions so CRSS implemented in ABAKO was used. This map provides valuable information since it identifies the most abundant ions for each plasma conditions and thus allowing one to decrease the number of ions to be considered. This reduction allows us to include more atomic configurations per ion, which is important in the determination of the radiative properties. Another map of interest provides the thermodynamic regimes of the plasma as a function of matter density and electron temperature, since the resolution of SB equations is considerably easier than rate equations with a resulting reduction in computing time. To obtain this map, we proceeded as follows: when the ion populations, $p_{i}$, calculated from SB equations, $p_{i}^{\mathrm{SB}}$, present a mean deviation $(\Delta p)$, with respect to those obtained from the CRSS model, $p_{i}^{\text {CRSS }}$, is smaller than a certain criterion imposed $\left(\Delta p^{*}\right)$ then we consider that the use of LTE is acceptable for obtaining the ionization balance. The mean deviation is calculated as

$\Delta p^{\mathrm{LTE}}=\sqrt{\frac{\sum_{i}\left(p_{i}^{\mathrm{CRSS}}-p_{i}^{\mathrm{LTE}}\right)^{2}}{\sum_{i}\left(p_{i}^{\mathrm{LTE}}\right)^{2}}} \leq \Delta p^{*}$

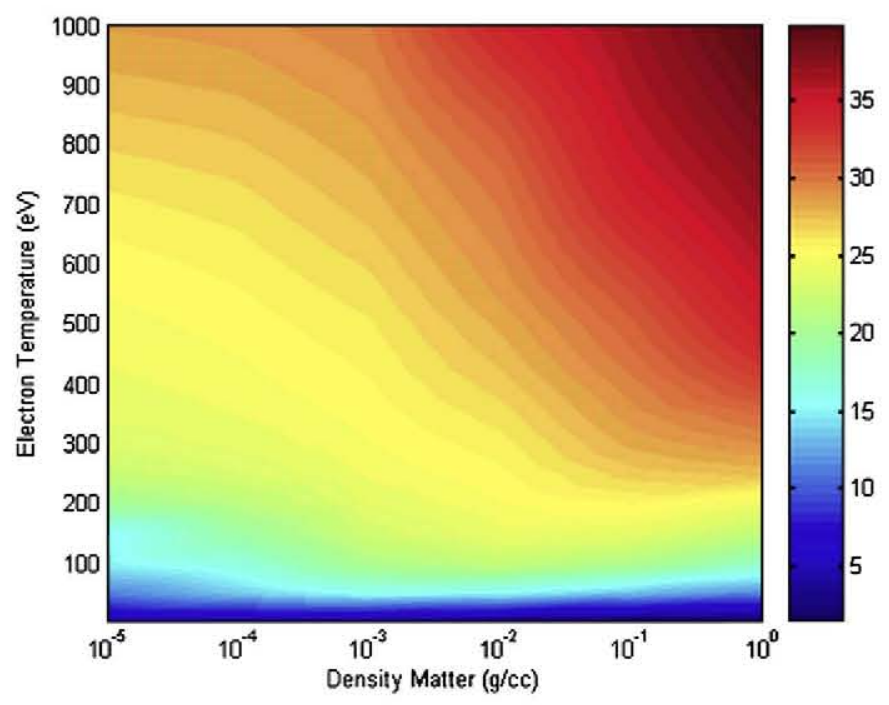

Fig. 1. Average ionization as a function of matter density and electron temperature.

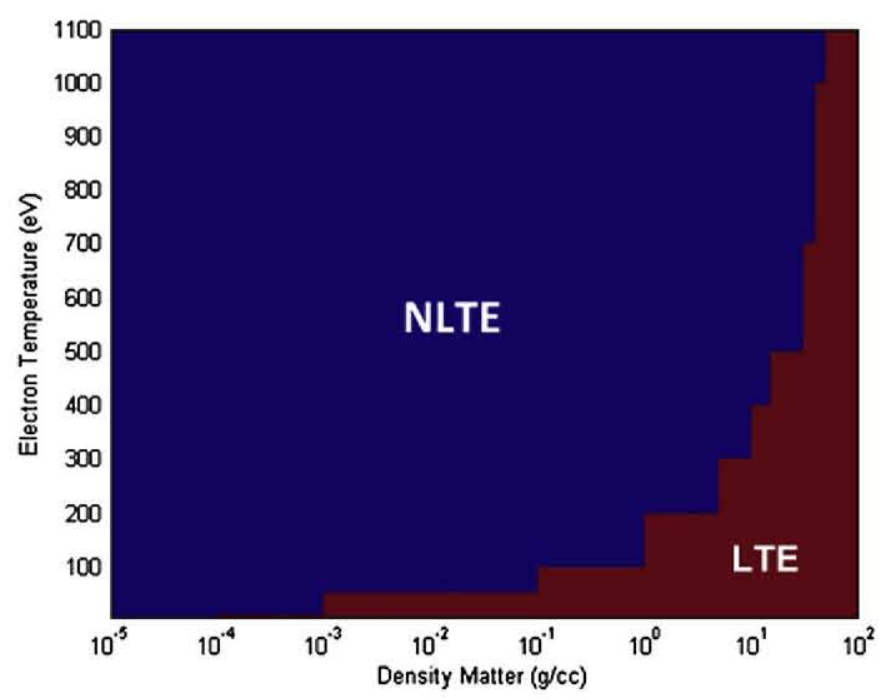

Fig. 2. Thermodynamic regimes.

where $i$ runs over the whole set of ions included in the calculations. In previous works $[27,39]$ the criterion, $\Delta p^{*}$, was fixed to $0.1(=10 \%)$ for the thermodynamic regime maps of carbon and aluminum and in this work for xenon we have kept the same value for the criterion. Accordingly, we obtained the thermodyanmic map for xenon plotted in Fig. 2. In Ref. [39] we proved that taking the value of the criterion equal to 0.1 ensures that the deviations in the average ionization were always less than or equal to $1 \%$. We have checked that this result also holds for xenon and we have also obtained that when the criterion is fulfilled the deviations for the Rosseland and Planck mean opacities are lower than $10 \%$ as it can be seen in Table 1. However, we have found that for the radiative power loss we can obtain deviations larger than $10 \%$, as can be observed in Table 1 for the case of $150 \mathrm{eV}$ and $0.5 \mathrm{gcm}^{-3}$. According to Fig. 2, this case should be LTE but it is located in the border between LTE and NLTE regimes. In such situations the ground and the first excited levels could be in LTE but not the more excited ones. This fact is well illustrated in Fig. 3, where we have shown the multifrequency opacity and emissivity for this particular case. From the figure we observe good agreement between the SB and the CRSS calculations of the multifrequency opacity whereas for the emissivities some differences are observed, which explains the low deviations in the mean opacities and the large discrepancies in the radiative power loss. On the other hand, in our analysis we have found that for regions far from the LTE regime, the SB calculations for the average ionization can be overestimated more than $100 \%$ with respect to the values provided by the CRSS model. Thus, for example, for a temperature of $200 \mathrm{eV}$ and matter density of $10^{-5} \mathrm{gcm}^{-3}$ we obtained $\bar{Z}_{S B}=40.97$ and $\bar{Z}_{\mathrm{CRSS}}=19.89$. In the mean opacities and radiative power losses these discrepancies can reach two orders of magnitude, which seen in Fig. 4. From the figure we can

Table 1

Analysis of the mean deviations for the average ionization $(Z)$, charge state distribution ( $p$ ), Planck and Rosseland mean opacities (PMO and RMO, respectively) and radiative power loss (RPL).

\begin{tabular}{lcllcll}
\hline $\mathrm{T}(\mathrm{eV})$ & $\rho\left(\mathrm{gcm}^{-3}\right)$ & $\Delta \mathrm{Z}(\%)$ & $\Delta \mathrm{p}(\%)$ & $\Delta \mathrm{RPL}(\%)$ & $\Delta \mathrm{PMO}(\%)$ & $\Delta \mathrm{RMO}(\%)$ \\
\hline 10 & $10^{-3}$ & 0.50 & 1.28 & 9.93 & 4.46 & 1.81 \\
150 & 0.5 & 0.58 & 7.90 & 13.60 & 1.10 & 0.60 \\
200 & 5 & 0.31 & 1.93 & 3.48 & 0.18 & 0.47 \\
500 & 30 & 0.48 & 2.55 & 2.36 & 1.16 & 1.41 \\
750 & 40 & 0.63 & 2.73 & 3.45 & 1.49 & 0.92 \\
1000 & 50 & 0.46 & 2.64 & 4.15 & 6.71 & 2.81 \\
\hline
\end{tabular}



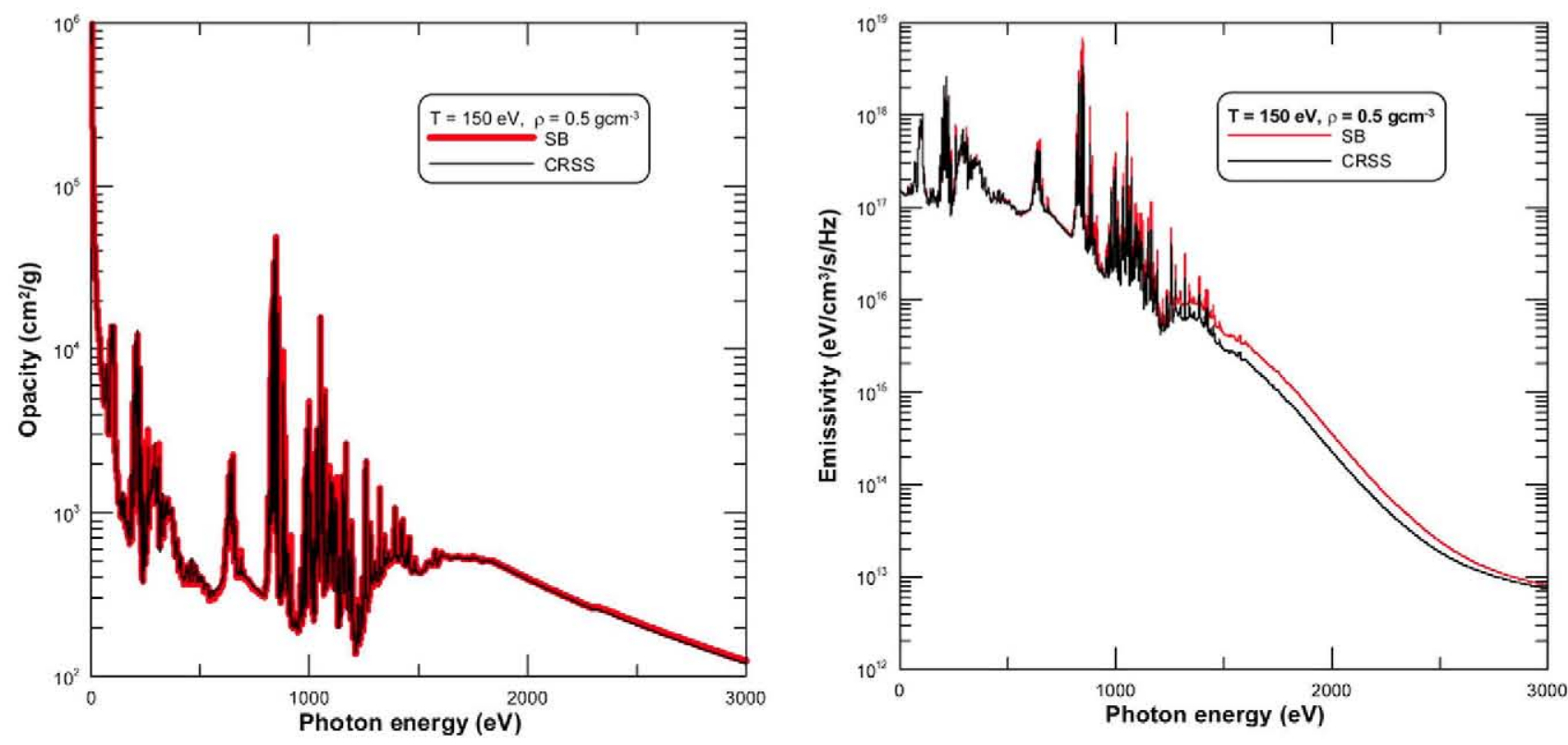

Fig. 3. Comparison between CRSS and SB calculations of the multifrequency opacity (left) and emissivity (right).

also observe that for regions in the NLTE regime, the SB model overestimates the radiative power loss and underestimates the mean opacities.

\subsection{Characterization of blast waves launched in xenon clusters}

We now apply the results above to characterizing radiative blast waves launched in xenon clusters. In Ref. [40] the conditions to define an optically thin, radiative regime for laboratory astrophysics experiments relevant to supernova remnants were formulated. In particular, in order to be optically thin the radiative flux should escape from the blast wave which implies that the photon mean free path, $\lambda_{\text {rad }}$, must be larger than a characteristic size scale of the system, $h$. Furthermore, to be radiative, the radiative cooling time, $\tau_{\text {rad, }}$ must be shorter than the convective

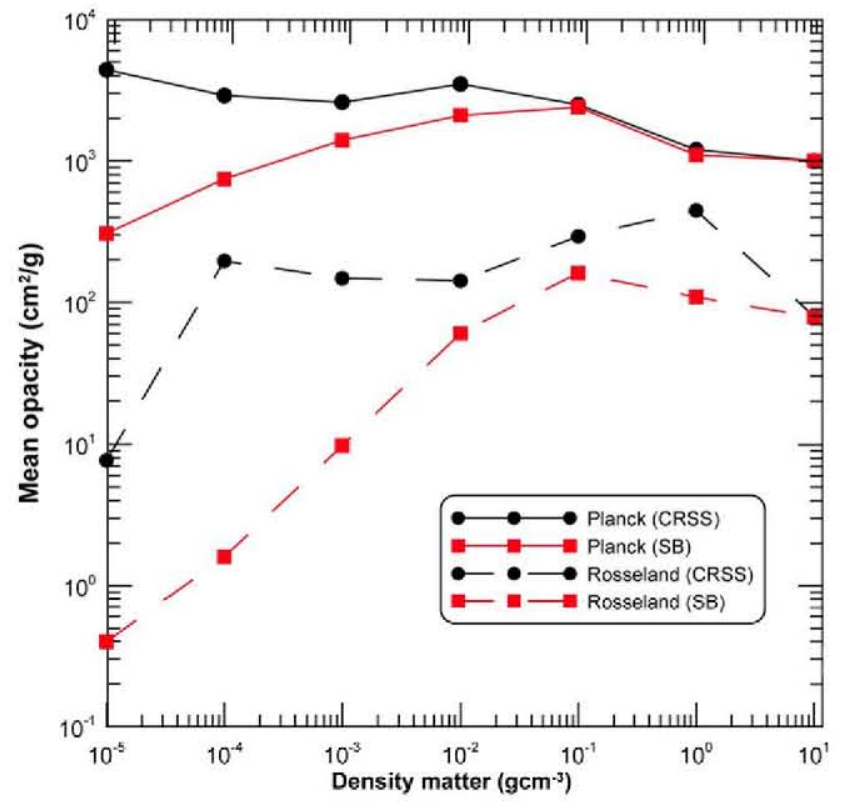

transport time, $\tau_{\text {conv }}=h / s$, with $s$ the plasma sound speed. If the plasma is optically thin the radiative cooling time is evaluated as [40]

$\tau_{\text {rad }}=2.4 \times 10^{-2} \frac{(Z+1) T}{Z n_{i} \Lambda}$

where $T$ is the electron temperature in $\mathrm{eV}, n_{i}$ is the ion density in $\mathrm{cm}^{-3}$ and $A$ is the cooling function in ergs $\mathrm{cm}^{3} \mathrm{~s}^{-1}$, which is the radiative power loss divided by the electron and ion particle densities. For optically thick plasmas, $A$ should be replaced by the radiative flux [41]. In Ref. [14] an analysis was carried out of blast waves launched in clusters that have typical gas densities around $5 \times 10^{-4} \mathrm{gcm}^{-3}$ and post-shock temperatures around 5-10 eV and, according to our calculations of the average ionization shown in Fig. 1, average ionizations between 3.5 and 6.5. For $h=0.01 \mathrm{~cm}$ Symes et al. concluded that the plasma was optically thin and

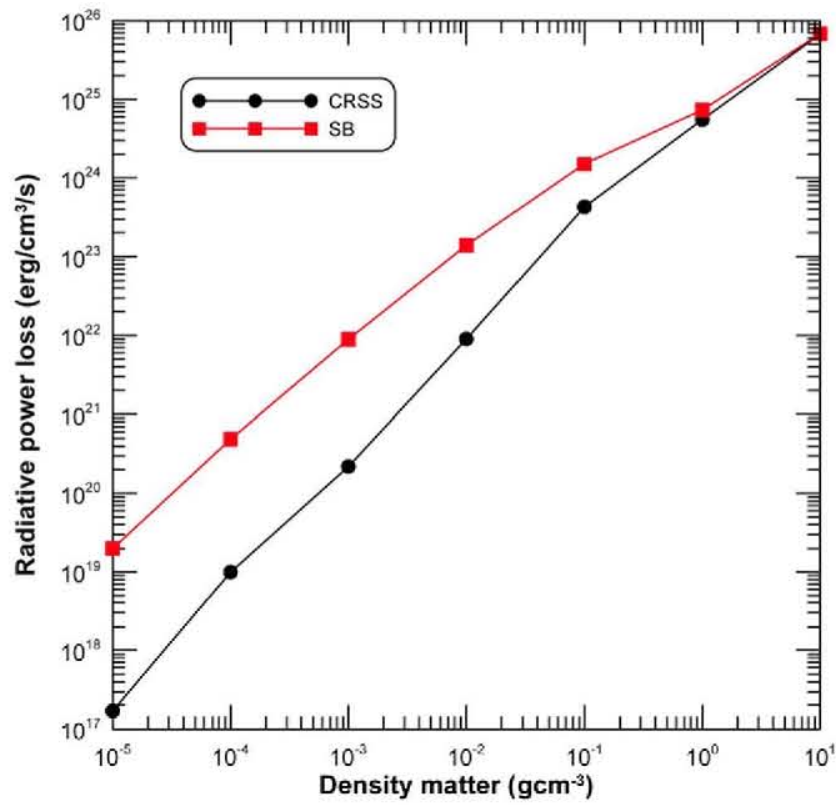

Fig. 4. Comparison between CRSS and SB calculations of the Planck and Rosseland mean opacities (left) and radiative power losses (right) for a temperature of $200 \mathrm{eV}$. 


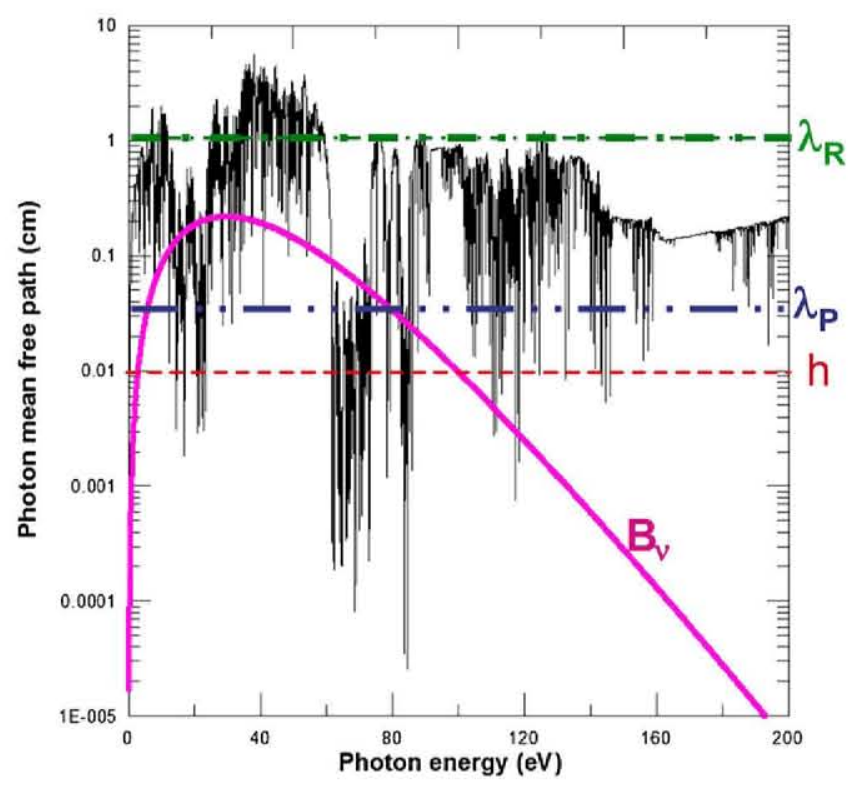

Fig. 5. Analysis of the multifrequential, Rosseland $\left(\lambda_{R}\right)$ and Planck $\left(\lambda_{P}\right)$ mean free paths. $h$ and $B_{v}$ denote the characteristic size and the Planck function, respectively.

Table 2

Analysis of the mean deviations for the average ionization $(Z)$, charge state distribution $(p)$, Planck and Rosseland mean opacities (PMO and RMO, respectively) and radiative power loss (RPL) for the pre- and post-shock regions.

\begin{tabular}{lcc}
\hline Mean deviation\% & Pre-shock & Post-shock \\
\hline$\Delta Z$ & 0.83 & 0.78 \\
$\Delta \mathrm{p}$ & 5.00 & 4.63 \\
$\Delta$ PMO & 6.45 & 5.00 \\
$\Delta$ RMO & 3.83 & 2.76 \\
$\Delta$ RPL & 28.50 & 15.52 \\
\hline
\end{tabular}

radiative. However, their calculation of the mean free path only included the bremsstrahlung contribution to the opacity that is not a good approximation at these low densities and temperatures where line radiation is dominant. On the other hand, their calculation of the cooling time was made using the cooling function

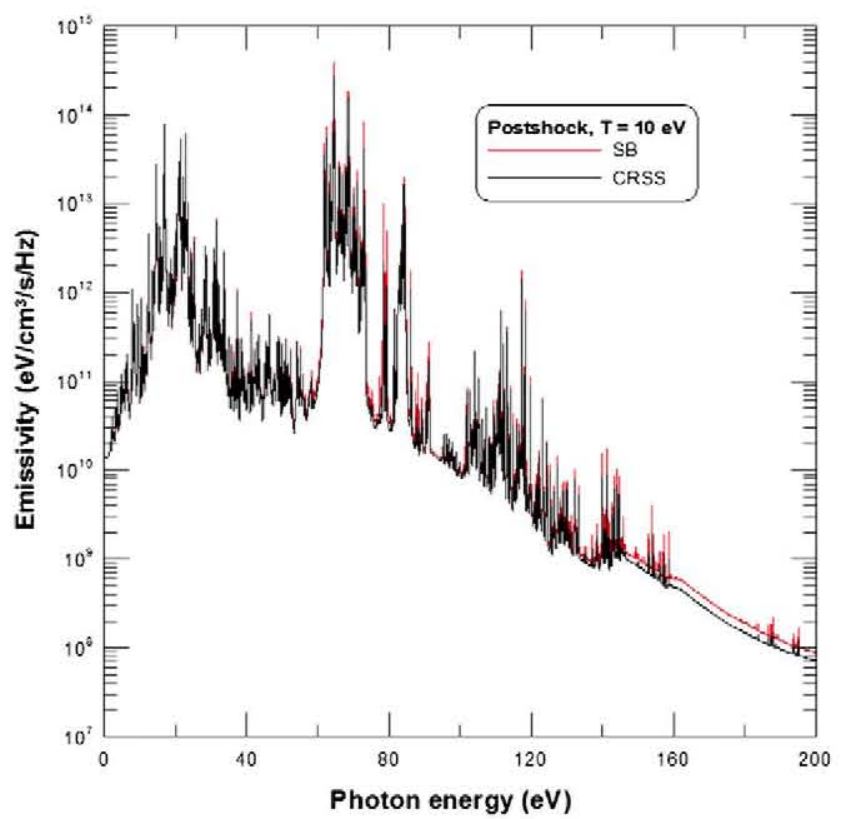

tabulated in Ref. [42], which solves a coronal equilibrium model to obtain the level populations and is accurate for electron densities lower than those obtained in the experiments of interest here.

Using RAPCAL we have calculated both the Rosseland and Planck mean free paths including the bound-bound and bound-free contribution. From our calculations we find that the region where the plasma can be assumed optically thin is shifted, for a given temperature, toward lower densities due to the increases the opacity from line absorption. For the typical density of $5 \times 10^{-4} \mathrm{gcm}^{-3}$ and temperature of $5 \mathrm{eV}$ the Rosseland and Planck mean free paths obtained were 0.21 and $0.019 \mathrm{~cm}$, respectively, and for a temperature of $10 \mathrm{eV}$, these were 0.74 and $0.03 \mathrm{~cm}$, respectively. All of the mean free paths calculated are larger than $h$ and, therefore, we conclude that the plasma in the post-shock region is optically thin. Thus, Planck averaging is justified and commonly used [43]. The results yield Planck mean free paths that are of the same order than $h$, and, therefore, we have analyzed the multifrequency opacity in order to detect in which spectral range the plasma is not optically thin. In Fig. 5 we show the spectrally resolved, Planck and Rosseland mean free paths and the characteristic size of the plasma for the temperature of $10 \mathrm{eV}$. Further, we have also plotted the Planck function to show which spectral ranges present a higher contribution in the Planck mean opacity. From the figure we observe that the plasma is optically thick only for the photons in the energy range of $60-85 \mathrm{eV}$, so for almost the whole photon energy range the plasma can be assumed as optically thin. Therefore, we can employ Eq. (2) to determine if the blast wave is in the radiative regime.

From our calculations we find that for the density of $5 \times 10^{-4} \mathrm{gcm}^{-3}$ the plasma enters in the radiative regime for temperatures lower than $\approx 300 \mathrm{eV}$. From the calculations using the cooling functions provided in Ref. [42], the temperature is around $600 \mathrm{eV}$. The cooling functions given in Ref. [42] were calculated for electron densities lower than $10^{16} \mathrm{~cm}^{-3}$, whereas the electron density in this case is $\approx 10^{19} \mathrm{~cm}^{-3}$ and, therefore, their results in this case could not be accurate. In any case, RAPCAL results also predicts that the blast waves under analyses are in the radiative regime.

Finally, we have analyzed the thermodynamic regime in this kind of experiments. For this purpose, we have analyzed a shock launched in xenon using the THOR laser system at the University of

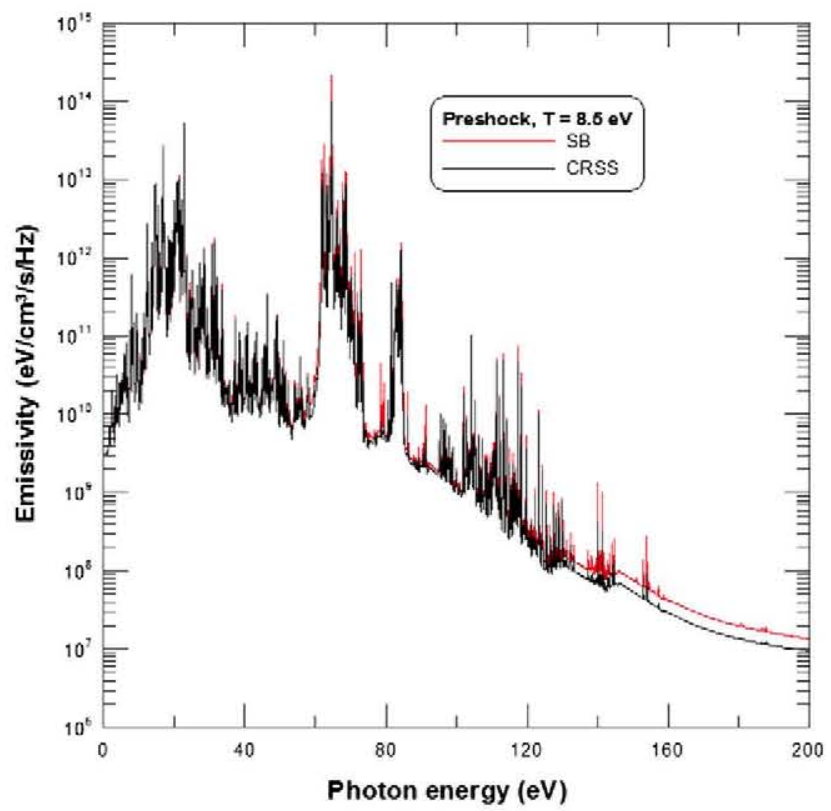

Fig. 6. Comparison between CRSS and SB emissivities for the post-shock (left) and pre-shocks (right) regions. 
Texas [12]. The gas density is $0.247 \mathrm{~kg} \mathrm{~m}^{-3}$ and the electron density and average ionization of the pre- and post-shock regions are known. However, the electron temperature is unknown and in Ref. [14] it was estimated assuming LTE and applying an approach described in Ref. [44]. We have estimated the temperature solving both SB equations and the CRSS model. At $17 \mathrm{~ns}$, for the pre-shock region the temperature given in Ref. [14] is around $7.5 \mathrm{eV}$, whereas our SB and CRSS calculations provided temperatures of 8.5 and $8.6 \mathrm{eV}$, respectively. For the post-shock region the temperature given by Ref. [14] is around $8.5 \mathrm{eV}$ whereas our SB and CRSS estimation of the temperature are around 10 and $10.1 \mathrm{eV}$, respectively. First, from the results we observe that the LTE estimate from Ref. [14] gives a temperature which is around $1 \mathrm{eV}$ lower than the value given by the SB equations. On the other hand, we detect that the SB and CRSS models provide very similar temperatures. In Table 2 we present the mean deviations between SB and CRSS calculations both for the pre- and post-shock regions. From the table, and according to the criterion presented, we could assume that the plasma is in the LTE regime. However, these cases of density and temperatures are near the border between the LTE and NLTE regimes, and, therefore, the discrepancies in the radiative power loss larger than $10 \%$ are expected due to the excited states could be in NLTE. This fact can be seen in Fig. 6 where noticeable differences between SB and CRSS emissivities are observed. Therefore, for these cases, although the SB equations would provide acceptable values of average ionization or mean opacities, the results for the radiative power loss and the emissivity would be not accurate enough and since we are dealing with radiative shocks, where the emissivity plays a pivotal role, a CRSS calculation would be required.

\section{Conclusions}

Xenon is commonly used for laboratory astrophysics simulations, in particular to study radiative shocks. In these cases, plasma radiative properties are key quantities and therefore should be calculated accurately. Since xenon a high $\mathrm{Z}$ element, the determination of its radiative properties is a complex task under NLTE conditions, and, therefore, theoretical studies are needed. In this work, making use of the CRSS model implemented in the computational package ABAKO/RAPCAL, we have presented maps of the average ionization and thermodynamic regimes of xenon for a wide range of plasma densities and temperatures. These maps provide useful information and help to optimize subsequent calculations. For obtaining the thermodynamic regimes, we have employed the criterion that LTE regime is attained when the mean deviation between CRSS and SB calculations of the charge state distribution is lower than $10 \%$. We have checked that when the criterion is fulfilled the mean deviation in the average ionization is lower than $1 \%$ and lower than $10 \%$ in Rosseland and Planck mean opacities, and it is also found that good agreement occurs for the multifrequency opacities. However, for the regions near the border between NLTE and LTE regimes, although the criterion is fulfilled, the differences in the emissivity and radiative power loss can be larger than $10 \%$ since many excited levels can be in NLTE. Therefore, when we are interested in the emissivity or radiative power loss, the criterion must be employed with care.

We have applied our study to characterize typical experiments of radiative blast waves launched in xenon clusters. Due to the low temperature, xenon ionization is also low, and, therefore, radiative properties are dominated by line emission and calculations including only bremssthralung contribution provide a poor approach and overestimate the mean free path. On the other hand, the cooling functions provided by Ref. [42] were calculated for low electron densities $\left(<10^{16} \mathrm{~cm}^{-3}\right)$ and are not appropriate for the densities reached in these experiments, which are three orders of magnitude higher. With RAPCAL we have shown that the blast waves launched in xenon clusters are in the radiative regime and belong to the optically thin-thin regime of the radiative shocks, although the plasma is optically thick for some photon energy ranges. Finally, we have determined that to obtain accurate values of the emissivity and radiative power loss, key quantities in the study of the radiative blast waves, these should be calculated using the CRSS model even though in the plasma conditions under analysis the $\mathrm{SB}$ equations results are similar to those derived from the CRSS approach for the average ionization or the mean opacities.

\section{Acknowledgments}

This work has been supported by the Research Project of the Spanish Government (ENE2009-11208/FTN), by the Research Project of the Government of the Canary Islands (SolSubC200801000057) and also by the Keep in touch Project of the European Union.

\section{References}

[1] A.S. Moore, J. Lazarus, M. Hohenberger, et al., Astrophys. Space Sci. 307 (2007) 139

[2] T. Ditmire, K. Shigemori, B.A. Remington, et al., Astrophys. J. Suppl. Ser. 127 (2000) 299.

[3] R.P. Drake, A.B. Reighard, Proc Conf Aps Topical Group on Shock Compression of Condensed Matter Cp845, 1417 (2006).

[4] M. Gonzalez, C. Stehle, E. Audit, et al., Laser Part Beams. 24 (2006) 535.

[5] M. Busquet, E. Audit, M. Gonzalez, C. Stehle, et al., High Energy Density Phys. 3 (2007) 8

[6] C. Michaut, E. Falize, C. Cavet, et al., J. Phys. Conf. Ser. 112 (2008) 042013.

[7] C. Stehle, A. Ciardi, J.P. Colombier, et al., Laser Part. Beams. 27 (2009) 709.

[8] J. Lindl, Phys. Plasmas 2 (1995) 3933.

[9] X. Fleury, S. Bouquet, C. Stehle, et al., Laser Part Beams. 20 (2002) 263.

[10] S. Bouquet, C. Stehle, M. Koenig, et al., Phys. Rev. Lett. 92 (2004) 5001

[11] A.B. Reighard, R.P. Drake, J.E. Mucino, et al., Phys. Plasmas 14 (2007) 056504.

[12] J. Osterhoff, D.R. Symes, A.D. Edens, et al., New J. Phys. 11 (2009) 023022.

[13] C. Stehle, M. Gonzalez, M. Kozlova, et al., Laser Part Beams. 28 (2010) 253.

[14] D.R. Symes, M. Hohenberger, J. Lazarus, et al., High Energy Density Phys. 6 (2010) 274.

[15] O. Peyrusse, Nucl. Fusion 44 (2004) 5202.

[16] D.R. Bates, A.E. Kingston, R.W.P. McWhirter, Proc. R. Soc. London Ser. A 267 (1962) 297.

[17] A. Bar-Shalom, J. Oreg, M. Klapisch, J. Quant. Spectrosc. Radiat. Transfer 58 (1997) 427.

[18] O. Peyrusse, J. Quant. Spectrosc. Radiat. Transfer 71 (2001) 571.

[19] J. Bauche C Bauche-Arnoult, M. Klapisch, Adv At Mol Phys. 23 (1987) 131.

[20] A. Bar-Shalom, J. Oreg, W.H. Goldstein, et al., Phys. Rev. A 40 (1989) 3183.

[21] S. Mazevet, J. Abdallah, J. Phys. B: At. Mol. Opt. Phys. 39 (2006) 3419.

[22] S. Hansen, J. Bauche, C. Bauche-Arnoult, et al., High Energy Density Phys. 3 (2007) 109 .

[23] J. Abdallah, M.E. Sherrill, D.P. Kilcrease, et al., High Energy Density Phys. 5 (2009) 204.

[24] R.P. Drake, Astrophys. Space Sci. 298 (2004) 49.

[25] R. Rodriguez, R. Florido, J.M. Gil, et al., Commun. Comput. Phys. 8 (2010) 185

[26] R. Florido, R. Rodriguez, J.M. Gil, et al., Phys. Rev. E 80 (2009) 056402.

[27] R. Rodriguez, R. Florido, J.M. Gil, et al., Laser Part Beams. 26 (2008) 433

[28] C. Bowen, R.W. Lee, Yu Ralchenko, J. Quant. Spectrosc. Radiat. Transfer 99 (2006) 102.

[29] J.G. Rubiano, R. Florido, C. Bowen, et al., High Energy Density Phys. 3 (2007) 225.

[30] C.J. Fontes, J. Abdallah, C. Bowen, et al., High Energy Density Phys. 5 (2009) 15.

[31] W. Lotz, Z. Phys. 206 (1968) 205

[32] H.V. Van Regemorter, Astrophys. J. 136 (1962) 906

[33] H.A. Kramers, Philos. Mag. Lett. 46 (1923) 836.

[34] H.R. Griem, Principles of Plasma Spectroscopy. Cambridge University Press, Cambridge, 1997, pp. 177-179.

[35] M.F. Gu, Can. J. Phys. 86 (2008) 675

[36] J.C. Stewart, K.D. Pyatt, Astrophys. J. 144 (1966) 1203

[37] O. Peyrusse, C. Bauche-Arnoult, J. Bauche, Phys. Plasmas 12 (2005) 063302

[38] M.S. Dimitrijevic, N. Konjevic, Astron. Astrophys. 172 (1987) 345.

[39] J.M. Gil, R. Rodriguez, R. Florido, et al., Laser Part Beams. 26 (2008) 21

[40] D. Ryutov, R.P. Drake, J. Kane, et al., Astrophys. J. 518 (1999) 821.

[41] C. Michaut, E. Falize, C. Cavet, et al., Astrophys. Space Sci. 322 (2009) 77

[42] D.E. Post, R.V. Jensen, C.B. Tarter, et al., Atom Data Nucl. Data 20 (1977) 397.

[43] M.R. Zaghlou, Plasma Phys. Control. Fusion. 49 (2007) 1129

[44] Y.B. Zel'dovich, Y.P. Raizer, Physics of Shock Waves and High-Temperature Hydrodynamic Phenomena. Dover, New York, 2002. 